\title{
Ein- und Ausschliessungspraktiken als Konstituierung von Grenzen
}

\author{
Catrin Heite $\cdot$ Marion Pomey $\cdot$ Charlotte Spellenberg
}

Zusammenfassung: Grenzen haben symbolische und soziale Bedeutung, reproduzieren durch moralisierende Zuschreibungen soziale Ungleichheiten und generieren Ein- und Ausschlüsse bestimmter Personen(gruppen). Grenzen bzw. eine grenzanalytische Perspektive sind für die Sozialpädagogik deshalb relevant, weil sie Aufschluss geben über normative Ordnungsvorstellungen sowie die eigene sozialpädagogische Beteiligung hieran. Normative Ordnungen ziehen Grenzen zwischen krisenhaftem Sein und idealem Sollen und bringen soziale Wirklichkeiten hervor. Durch so konstituierte Grenzverhältnisse von Wirklichkeit und Möglichkeit lassen sich auch empirisch Grenzziehungen rekonstruieren, die Ein- und Ausschlüsse hervorbringen. Dies wird im Artikel exemplarisch für die sozialpädagogische Handlungspraxis herausgearbeitet und normative Ordnungen von ,guter" und „schlechter" Elternschaft rekonstruiert. Eine grenzanalytische Perspektive macht damit (Un)Möglichkeiten des (Anders-)Seins als kontingente und normativ plausibilisierte Ausschließungen erkennbar und legt sozialpädagogische Wirklichkeitskonstruktionen samt ihrer Konsequenzen in ihrer Bearbeitbarkeit offen.

Schlüsselwörter: Grenzanalyse · symbolische und soziale Grenze · Soziale Ungleichheit · (normative) Ein-/Ausschlüsse · Konstituierungen von Wirklichkeit und Möglichkeit · (Hetero)normative Elternkonzepte

\section{Constitution of boundaries as practices of inclusion and exclusion}

\begin{abstract}
Boundaries have symbolic and social meaning, reproduce social inequalities via moralizing ascriptions and generate in- and exclusions of specific (groups of) persons. Boundaries resp. a boundary analytic perspective are relevant for Social Work in so far, as they shed light on normative conceptions of order and the own involvement of Social Work within these processes. Normative orders draw boundaries between a critical be and an ideal should-be, they create social realities. By these constituted boundary relations of reality and possibility, empiric
\end{abstract}

(C) Die Autor(en) 2013. Dieser Artikel ist auf Springerlink.com mit Open Access verfügbar.

Prof. Dr. C. Heite $(\bowtie) \cdot$ M. Pomey $\cdot$ C. Spellenberg Institut für Erziehungswissenschaft, Universität Zürich, Freiestraße 36, 8032 Zürich, Schweiz

E-Mail: c.heite@ife.uzh.ch

M. Pomey

E-Mail: mpomey@ife.uzh.ch

C. Spellenberg

E-Mail: c.spellenberg@ife.uzh.ch 
boundaries - which create in- and exclusions - can be reconstructed. In the article this is shown exemplarily for the socio-pedagogic practice and the normative order of ,good' and ,bad ' parenting. Therefore, a boundary analytic perspective makes (im)possibilities of being (different) recognizable as contingent and normatively plausibilized exclusions, it discloses socio-pedagogic constructions of reality with their consequences and workability.

Keywords: Boundary analysis · Social inequality · (Normative) Inclusion/exclusion · Constitutions of reality and possibility $\cdot($ Hetero)normative concepts of parenting

„Daher muß man“ - so analysiert Jacques Donzelot in Die Ordnung der Familie - ,in jedem Hilfeersuchen die moralische Verfehlung finden und aufdecken, die ihr mehr oder weniger direkt zugrunde liegt: diesen Anteil an Leichtsinn, Faulheit, Verkommenheit, der sich in allem Elend findet“" (Donzelot 1980, S. 82). Im Anschluss an diesen Blick auf die Bearbeitung des Sozialen - und damit auch auf moralisierende Zuschreibungen an die AdressatInnen der Sozialpädagogik - wird nun zunächst eine grenzanalytische Perspektive skizziert (1.), um damit Ein- und Ausschließungspraktiken als Konstituierung von Grenzen zu umgreifen. Darauf aufbauend wird zunächst der Zusammenhang von Grenzsetzungen und Normativität diskutiert (2.), um dies dann empirisch für das Feld der familialen Krisenintervention zu justieren (3.) ${ }^{1}$. Abschliessend wird der Begriff der Grenze als Analysekategorie von Grenzziehungsprozessen und in seiner Bedeutung für die Sozialpädagogik resümiert (4).

\section{Grenzanalytische Perspektive}

Mit dem analytischen Konzept der Grenze werden ganz unterschiedliche Fragestellungen wie etwa nationalstaatliche und räumliche Grenzen, kulturelle, politische, soziale Grenzen und in diesem Zusammenhang Macht und Herrschaft, soziale Ungleichheit, Normen, Diskurse, Wissen und Wissensproduktion bearbeitet.

Der folgenden Argumentation liegt eine Bestimmung von symbolischen und sozialen Grenzen nach Lamont und Molnár (2002) zugrunde:

Symbolic boundaries are conceptual distinctions made by social actors to categorize objects, people, practices, and even time and space. They are tools by which individuals and groups struggle over and come to agree upon definitions of reality. Examining them allows us to capture the dynamic dimensions of social relations, as groups compete in the production, diffusion, and institutionalization of alternative systems and principles of classifications. [...] They are an essential medium through which people acquire status and monopolize resources. Social boundaries are objectified forms of social differences manifested in unequal access to and unequal dis-

1 Diese Kapitel sind im Rahmen gemeinsamer Diskussionen entstanden und wurden als Einzelbeiträge auf der Kommissionstagung Sozialpädagogik 2013 in Tübingen präsentiert. Sie spiegeln die Forschungsschwerpunkte der Autorinnen wider und wurden daher vorwiegend einzeln erstellt: (1) Grenzanalytische Perspektive - Catrin Heite, (2) Normativität - Charlotte Spellenberg und (3) Grenze empirisch: Krisenintervention - Marion Pomey. 
tribution of resources (material and nonmaterial) and social opportunities. (Molnár und Lamont 2002, S. 168)

Symbolische Grenzen dienen demnach der Kategorisierung von Dingen, Menschen und Praktiken. Sie dienen zur Verständigung über das, was ist, was sein soll und was nicht sein soll. Also markieren symbolische Grenzen das Normale und das Abweichende, das Anerkennungsfähige und das Nicht-Anerkennungsfähige. Symbolische Grenzen werden diskursiv zum Beispiel mit Begriffen wie Leichtsinn, Faulheit, Verkommenheit gezogen. Solche symbolischen Grenzen sind normativ und lassen sich auch empirisch auffinden (vgl. Pkt. 2. und 3.).

Soziale Grenzen sind - folgt man der Bestimmung von Lamont und Molnár - die objektivierten oder materialisierten Konsequenzen symbolischer Grenzen, also etwa ungleicher Zugang zu materiellen Gütern und anderen wertgeschätzten Ressourcen wie Bildung, politischer Beteiligung und Formen der Lebensführung.

Analytisch unterstützt die Perspektive auf symbolische und soziale Grenzen die Untersuchung gesellschaftlicher Strukturen und sozialer Verhältnisse, da sie Ungleichheiten, Machtverhältnisse und soziale Positionen sowie (diskursive) Praktiken des Ein- und Ausschlusses in den Blick nimmt. Grenzen dienen so der Erfindung und Verteidigung von Hierarchien, sie strukturieren, verhindern, begrenzen und provozieren menschliches Handeln, schließen ein und aus. Als symbolische, territoriale, politische, normative und soziale Instanzen bringen sie quasi die Dinge ,ins Reine“:

D. h.: „die Grenze“ markiert soziale Ordnungsvorstellungen, Dualismen des Möglichen und des Unmöglichen, des Akzeptablen und Inakzeptablen, Normalen und Abweichenden, des Eigenen und Anderen, des Zugehörigen und Nicht-Zugehörigen: Die Grenze klassifiziert, sortiert, normiert, schließt ein und aus, sie privilegiert und deprivilegiert, weist Rechte zu und entzieht sie; die Grenze ist Ausdruck von Macht- und Herrschaftsverhältnissen und ein Mittel zu deren Erhaltung.

Ein Feld, in dem all dies eine Rolle spielt, ist „das Soziale“ und die „Soziale Frage“. Als Handlungsfeld der Sozialpädagogik - als Wissenschaft und als Profession - wird diese grenzanalytische Perspektive nun mit Blick auf die Grenzen des Sozialen und des Sozialpädagogischen skizziert. Denn durch die symbolische Grenze werden bestimmte Gruppen als ausserhalb des Normalen, Akzeptablen, Guten markiert und damit zu AdressatInnen der Sozialpädagogik gemacht.

Seit der erstmaligen Formulierung der Sozialen Frage im 19. Jahrhundert und der Erfindung von Techniken, Methoden und Verfahren ihrer Bearbeitung sind jene Begriffe, jene normativen Zuschreibungen präsent, die Jacques Donzelot mit Leichtsinn, Faulheit, Verkommenheit umgreift und die sich ergänzen lassen mit - historisch und aktuell virulenten - Begriffen und Zuschreibungen wie etwa Disziplinlosigkeit, Bildungsmangel, Verwahrlosung, mangelnder Aufstiegswille, Abhängigkeit von staatlichen Transferleistungen, problematischer Medienkonsum, Tabak, Alkohol und Lottospiel, mangelnde Erziehungskompetenz und Kindesvernachlässigung. (Zur Konstruktion von ,guter“" und „schlechter“ Elternschaft und entsprechenden normativen Zuschreibungen vgl. Pkt. 2. und 3.).

Das historische und aktuelle Sprechen über die Personengruppen, die den Kern der Sozialen Frage ausmachen - Erwerbslose, „schlechte Eltern“, „verhaltensauffällige Kinder“, „,deviante Jugendliche“, „bildungsferne Familien“, Menschen, die ,abhängig“ sind von staatlichen Transferleistungen - stellt diese Gruppen ausserhalb der Grenzen des 
Normalen. Dadurch, dass die Besprochenen sich an den Grenzen des Sozialen bewegen, werfen sie die Soziale Frage erst auf. Sie werden besprochen als Gruppe, deren Form der Lebensführung, deren moralische Verfasstheit, deren Charakter nicht den gesellschaftlichen Normen entspricht und die daher - unter anderem pädagogisch - wieder in die Grenzen des Sozialen hinein zu verschieben sind, indem etwa Erwerbslose aktiviert oder „deviante Jugendliche“ normalisiert werden und „schlechten“ Eltern Erziehungskompetenzen vermittelt werden.

Dies deshalb, weil sie als Bedrohung der gesellschaftlichen Ordnung, als Gefährdung des gesellschaftlichen Zusammenhalts gesehen werden und die daher jenem missachtenden und moralisierenden Sprechen - die Faulheit und Verderbtheit derjenigen, die sich ausserhalb der Grenzen akzeptabler Lebensführung befinden - unterzogen werden. Wie Robert Castel (2008) darlegt: Die Soziale Frage handelt von Prozessen der sozialen Disqualifizierung, Verwundbarkeit, Entkopplung und Missachtung. Als hierfür zentrale Grenzsetzung analysiert Castel die Unterscheidung von arbeitsfähigen und nicht arbeitsfähigen Armen. Diese Unterscheidung, diese Grenzsetzung zwischen guten und schlechten Armen verschiebt Personen, die arbeiten können, dies aber nicht tun, an die Grenzen des Sozialen. Hier flottieren sie an den symbolischen Grenzen, die über Sesshaftigkeit, Eingebunden-Sein in primäre Sozialbeziehungen und Teilnahme an Erwerbsarbeit markiert werden.

Das heisst: historisch der arbeitsfähige Bettler und aktuell Menschen, denen mangelnde Employability zugeschrieben wird, befinden sich an diesen Grenzen: Sie arbeiten nicht, sind nicht oder nur unzureichend subsidiär über primäre Sozialbeziehungen sesshaft versorgt, sondern sie betteln oder beziehen - unter Umständen „missbräuchlich“ angeblich zu grosszügige, passivierende Sozialleistungen.

Die symbolischen Grenzen, die mit den Begriffen Sesshaftigkeit/Territorialität, primäre Sozialbeziehungen, Subsidiarität, informelle Solidargemeinschaften sowie Erwerbsarbeit, Fleiss, Disziplin oder neu: Employability - die damit benannten symbolischen Grenzen stellen all jene, die diesen normativen Sollens-Aussagen nicht entsprechen, ins Abseits, markieren ihre Lebensführung normativ als inakzeptabel, als unnormal und machen sie zu Gruppen, die es auszumerzen, zu pädagogisieren, zu disziplinieren, zu moralisieren, zu normalisieren gilt.

Den an den Grenzen der Gesellschaft lebenden Personen und Klassen - historisch waren das die Vagabunden, das arbeitende und das arbeitslose Industrieproletariat, heute sind es Jugendliche, denen es an Employability mangelt oder Eltern, deren Erziehungskompetenzen als nicht hinreichend beurteilt werden - diesen Personen wird mit helfenden, unterstützenden, reintegrierenden und zugleich repressiven, moralisierenden, und disziplinierenden Maßnahmen begegnet. Hier ist also von Personen die Rede, deren Unterstützungsbedarf nicht - oder zumindest nicht primär - als materiell, sondern als moralisch besprochen wird. Es handelt sich in dieser Denkweise also vor allem um ein Problem des Charakters und der Lebensführung oder der Lebenseinstellung und fällt damit interventionslogisch in den Bereich pädagogischer Massnahmen. Dieses Sprechen über die AdressatInnen der Sozialpädagogik zeigt sich als Veräußerung von normativen Grenzziehungen, vermittels derer sozialpädagogische Theorien Wirklichkeiten als krisenhaft sowie Seinsweisen als problematisch hervorbringen. 


\section{Normativität}

Normativität hat in den sozialpädagogischen, ein- und ausgrenzenden Sollens-Ansprüchen sowie in dem Zustandekommen von Übereinstimmungen über ,definitions of reality“ (Lamont und Molnár 2002, S. 168) eine spezifische Bedeutung. Wird die Grenze primär in ihren verbindenden und weniger in ihren trennenden Eigenschaften fokussiert und ihre Verschiebbarkeit relevant gesetzt, zeigt sich, wie erst durch den theoretischen Zugriff sowie durch die Verwicklung mit Normativität Wirklichkeit als ein So-Sein hervorgebracht wird: Die Postulierung eines So-Seins beinhaltet ein Grenzverhältnis von Wirklichkeit und Möglichkeit, welches beide Seiten miteinander verknüpft.

Ein solcherart gefasster Grenzbegriff unterscheidet sich dann von dem Begriff der Differenz dahingehend, dass mit der Grenze die Verwobenheiten statt die Abgrenzung des „Anderen“ in den Blick genommen werden können. Ein Grenzverhältnis kann dann nicht in eine Differenz - mit eindeutig voneinander abzugrenzenden, quasi fixen Polen - aufgelöst werden: Die Bestimmung von Wirklichkeit verweist stets auf eine Nicht-Wirklichkeit. Vermittels dieses Grenzverhältnisses und unter Bezug auf normative Ordnungen wird über unterschiedliche Möglichkeiten anerkennungsfähiger Seinsweisen verhandelt.

Normative Ordnungen bringen Grenzen hervor, ${ }^{2}$ indem sie beispielsweise bestimmte Seinsweisen als (il)legitim markieren und plausibilisieren. Damit lässt sich das Normative dieser Grenzziehungen, dieser Ein- und Ausschließungspraktiken von Seinsmöglichkeiten, über Plausibilisierungsstrategien erfassen, in denen Normativität mit Forst und Günther als eine „Art der Bindung ohne Fessel“ (2011, S. 11) wirksam wird und plausible Gründe liefert ${ }^{3}$. „Normative Ordnungen ruhen basalen Rechtfertigungen auf und dienen entsprechend der Rechtfertigung von sozialen Regeln, Normen und Institutionen; sie begründen Ansprüche auf Herrschaft und eine bestimmte Verteilung von Gütern und Lebenschancen“ (Forst und Günther 2011, S. 11). Aus diesem rechtfertigenden Bezug gewinnen normative Gründe ihren Geltungs- und Verbindlichkeitsanspruch. Das Normative an symbolischen bzw. sozialen Grenzen ist dann die Art und Weise, wie diese mit Verbindlichkeit ausgestattete Gründe liefern, die anschlussfähig und anerkennbar erscheinen.

Eine wesentliche Bedeutung von Normativität zeigt sich darin, dass rechtfertigende Gründe innerhalb einer normativen Ordnung wie eine Tatsache erscheinen, wie ein „Sachverhalt, dessen Existenz man hinnimmt, aber nicht in Frage stellt“ (Forst und Gün-

2 Das Verhältnis von normativen Ordnungen, Grenzen und der Rolle der Sozialpädagogik ist dabei verschränkt zu denken: Zum einen bringen normative Ordnungen Grenzen hervor. So lassen sich pädagogische Entwürfe in Bezugnahme auf Normativität plausibilisieren. Die normativen Ordnungen fungieren damit als Rechtfertigungen, indem sie sozialpädagogischen Wirklichkeitsentwürfen dazu verhelfen, ihre Gültigkeit zu erlangen. Zum anderen bringt die Sozialpädagogik mit ihren Grenzziehungen zwischen Sein und Soll normative Ordnungen auch selbst hervor.

3 „Wenn Normativität auf diese Weise mit Gründen zu tun hat, dann können Normen als Prinzipien verstanden werden, die in substanzieller Weise angeben, welche Gründe Menschen haben, etwas zu glauben und zu tun" (Wallace 2011, S. 34). Epistemische Normen geben dann vor, was als Evidenz für Wahrheit zu gelten habe, praktische Normen, welche Gründe Handelnde für bestimmte Handlungen haben. 
ther 2011, S. 18). Der konstruktive und bestreitbare Charakter von Gründen ist dann kaum noch wahrnehmbar. So gibt es bestimmte Handlungszusammenhänge, die mit ziemlich großer Aussicht auf intersubjektive Übereinstimmung als sozialpädagogisch markiert werden können. Übereinstimmung darüber, dass in diesem Handlungskontext ein sozialpädagogisch bearbeitbarer Problemgehalt steckt, dass man ihn als sozialpädagogisch wahrnehmen kann, bzw. wahrnehmen sollte (vgl. Schäfer 1989). Es ist also ein normativer Horizont, eine Rechtfertigungsordnung, was hier wirksam wird, was intersubjektive Übereinstimmung wahrscheinlich macht, indem es über Normativität an plausible und verbindliche Gründe bindet.

Durch Tatsachen behauptende, normative Zugriffe werden Ungleichheiten über Ein- und Ausschließungen (re)produziert. Das, was ein- und ausgeschlossen wird, sind beispielsweise bestimmte Lebensweisen, als illegitim markierte Seins- und Verhaltensformen und Möglichkeiten des Anders-Seins. Über die Verwicklung mit Normativität wird verschleiert, wie ein Setzen von Grenzen an die kontingente Regulierung dessen geknüpft ist, was als eine intelligible Seinsweise produziert wird und was nicht (vgl. Butler 1995).

Die Rolle der Sozialpädagogik zeigt sich hier darin, dass sie in ihrem theoretischen Zugriff auf Wirklichkeiten die Grenzen der Sicht- und Sagbarkeiten von Seinsweisen bearbeitet sowie auch die Grenzen dessen gestaltet, welche Seinsweisen als problematisch zu markieren und welche Veränderungen realisierbar sind bzw. sein sollten. Dabei macht es einen Unterschied, ob in diesem Zusammenhang von Sein und Soll im Sinne eines Differenzverhältnisses gedacht wird, oder ob Wirklichkeit und Möglichkeit als in einem Grenzverhältnis miteinander verschränkt verstanden werden.

Sozialpädagogik bearbeitet das problematisierte Verhältnis von Sein und Sollen. Innerhalb ihrer Rekonstruktionen dieses Verhältnisses tendiert sie dazu, reaktiv zu argumentieren, indem sie bspw. Krisen als gegebene Tatsachen unterstellt. Solche, wie Dollinger (2008) formuliert, Zeitdiagnosen statten sozialpädagogische Interventionen im gelingenden Fall mit Legitimität aus - und eben dieses Gelingen, im Sinne der Herstellung von intersubjektiver Übereinstimmung, ist maßgeblich abhängig von Bezugnahmen auf normative Ordnungen.

Während das Auflösen von Begrenzungen, zwischen dem was ist und dem was sein könnte, zwar als Qualitätsbedingung der Sozialpädagogik gilt, könnte aber der Eindruck entstehen, dass diese von einem unbeteiligten, ausserhalb liegenden Ort aus agiert, von wo aus sie ein sozialpädagogisches Gegen-Wissen oder Gegen-Handeln zur Verfügung stellen kann. Verschleiert wird so die Perspektive auf die Sozialpädagogik, die ein- und ausschliesst, als Beteiligte an Grenzziehungen, ihre Rolle in der diskursiven Konstitution sozialer Lebensbedingungen: Denn Sozialpädagogik ist eben nicht ohne eigene normative Interessen und ihre Wirkmächtigkeit geht über das bloße Explizieren von Problemen und angemessenen Interventionsmöglichkeiten hinaus.

Wenn objektivistische Krisenunterstellungen die argumentative Basis für sozialpädagogische Forderungen bilden, wird dabei eine polarisierende Differenz zwischen einem krisenhaften Sein und einem idealen Sollen hervorgebracht. Aufgrund ihrer Normativität ermöglichen solche Differenzierungen plausibles Sein und Sollen zu behaupten: Hier das als problematisch markierte und so sozialpädagogisch relevante Sein; dort das legitimer- und plausiblerweise anzustrebende Sollen. Sein und Sollen werden hier als fixe Pole 
affirmativ vorausgesetzt, obwohl sie aus Ein- und Ausschließungspraktiken hervorgehen und aus diskurskonstruktivistischer Perspektive nicht rein bestimmt werden können. Es kann ohnehin auf kein objektives Kriterium für die theoretische Geltung eines sozialpädagogischen Zugriffs auf die Realität zurückgegriffen werden. Das, was sozialpädagogisch ist, wann ein sozialpädagogischer Handlungsbedarf vorliegt, ist keine Frage, die durch eine unabhängige empirische Beobachtung entschieden werden kann. Die Entwürfe sozialpädagogischer Wirklichkeit sind kontingent, relational und müssen gegen andere ausgewiesen werden - eingebunden in normative Rechtfertigungsordnungen werden sie verobjektiviert und plausibilisiert.

Würde man die Wirklichkeit gegenüber der Möglichkeit in dem Maße relevant setzen, dass diese wie eine legitime Grenze des Möglichen erscheint, würde in radikaler Weise über einen Rekurs auf das Mögliche das Sein in seinen Grenzen fixiert. Würde man umgekehrt, gegenüber einer vermeintlich eindeutigen Wirklichkeit die Möglichkeit relevant setzen, ,indem man auf die Offenheit der Zukunft verweist, darauf, dass auch die Gegenwart verschieden wahrgenommen werden kann, dass jeder Determinationsversuch durch Wirklichkeiten als solche dem Freiraum des Subjekts nicht gerecht werde" (Schäfer 2012, S. 670), würde man sich einer bodenlosen Kontingenz des Faktischen hingeben.

Es zeigt sich, dass das Sein im Verhältnis zum Sollen auf dem Spiel steht und dabei weder eine eigentliche Wirklichkeit noch die reine Möglichkeit in ihrer Wahrheit angerufen werden können, um diesen Konflikt vermittels eines vermeintlich eindeutigen Differenzverhältnisses aufzulösen. Stattdessen ließen sich Wirklichkeit und Möglichkeit als Grenzverhältnis fassen, und zwar - in Anlehnung an Alfred Schäfer (2012) - in der Art eines Möglichkeitsraums: So stehen sich beide nicht als differente Entitäten losgelöst gegenüber, sondern sind durch die Grenze miteinander verbunden. Mit Bezug auf einen solchen Möglichkeitsraum wird dann nicht eine oppositionelle Differenz relevant gesetzt, sondern gerade die Verwobenheit, durch die eindeutige Unterscheidungen nicht ohne weiteres zu treffen sind. Wenn Wirklichkeit nur gegenüber ihrer Möglichkeit, ihrer Kontingenz gefasst werden kann, steht dieses Grenzverhältnis für Möglichkeitsräume, in denen das Wirkliche immer nur eine Möglichkeit sein kann. Es steht für die „Befragbarkeit des Wirklichen“ (Schäfer 2012, S. 229), das als solches den Raum des Möglichen aber gleichsam realistisch begrenzt. Solche Begrenzungen zeigen sich in den ein- und ausschließenden symbolischen Grenzen insofern, als dass bestimmte Möglichkeiten, bestimmten Personen(gruppen) vorbehalten sind.

Ist sozialpädagogische Wirklichkeit vom theoretischen Zugriff und normativen Vorstellungen abhängig, ist das, was das sozialpädagogisch Bedeutsame einer Situation ist und was als ihre Beantwortung gilt, Gegenstand von Aushandlungs- und Produkt von Grenzziehungsprozessen. Seins- und Verhaltensweisen sind nicht per se und unstrittig problematisch und erfordern sozialpädagogischer Bearbeitung, sondern es bedarf normativer Grenzziehungen zur Begründung dieser Behauptung. Als Normatives hat das Sozialpädagogische damit konstitutive und ausgrenzende Bedeutung für den Zugriff auf Realität. Denn die Ebene der Gegenstandskonstitution, die Ebene des zu bearbeitenden Seins liegt in der Bestimmung dessen, was sozialpädagogisch Sinn macht: in der kategorialen Erfassung sozialpädagogischer Wirklichkeit. Mit dem Fokus auf Normativität heißt das: als interpretatorische Einheit von Definition und Rechtfertigung. 
Eine grenzanalytische Perspektive zeigt sich insofern als erkenntnisgewinnbringend, als das Polarisierungen aufgebrochen und ihre Verwobenheit sowie ganz spezifisch eben ihre Verwicklung in normative Rechtfertigungen rekonstruierbar werden. So ist in der Analyse an den Punkt zurückzukehren, an dem aus dem konstruierten Grenzverhältnis von Wirklichkeit und Möglichkeit polarisierende Differenzen gemacht werden, dann ist an den Punkt zurückzukehren, an dem normative und verobjektivierende Plausibilisierungen Tatsachen proklamieren. So ist nicht nur zu fragen, welche spezifische Art ein vorherrschendes krisenhaftes Sein aufweist und wie Abhilfe geschaffen werden kann, vielmehr ließe sich auch klären, welche Bedingungen, Interessen, Normalitätsaspirationen und Subjektverständnisse in diese Diagnostik mit einfließen. Dann wäre nicht nach richtiger oder falscher Realität bzw. ihrer Erfassung zu fragen, sondern nach ihrem Zustandekommen und der eigenen Rolle der Sozialpädagogik.

Mit der Grenze als Analysekategorie zeigt sich diese als verbindendes Element und steht gleichsam in ihrer Gestaltbarkeit für die Befragbarkeit des Wirklichen und Möglichen. Für die sozialpädagogische Erfassung von Ungleichheiten bedeutet das, dass es für die Analyse fruchtbar zu machen gilt, welche Grenzziehungen gesetzt oder genutzt wurden, um zu zuschreibenden Seinsaussagen zu kommen. Denn normativ begründende wie begründete Plausibilisierungen des gegenwärtig Sicht- und Sagbaren lassen sich als Begrenzungen des Seins fassen. Deshalb sind solche Plausibilisierungsstrategien immer wieder und immer neu zu rekonstruieren. Nichts muss sein, wie es ist und daher stellt sich die nicht final zu beantwortende Frage, wie es sein könnte oder sollte. Sozialpädagogische Antworten hierauf sind als normative Grenzziehungsprozesse zu verstehen, als Konstituierungen des Möglichkeitsraums - Konstituierungen, die als Schließung dieses Raums als Wirklichkeit immer provisorisch, prekär und veränderbar bleiben. Sozialpädagogische Theorie operiert dann nicht lediglich reaktiv in Bezug auf solche vorfindlichen Schließungsversuche. Sie ist sowohl selbst an solchen Schließungsversuchen beteiligt, sodass ihre Bearbeitungen von Ungleichheiten nicht außerhalb der jeweils gezogenen Grenzen situierbar sind. Und gleichsam ist es eine ihrer Aufgaben, diese Grenzen als verschiebbare offenzulegen und Verschiebungen möglich zu machen.

\section{Grenze empirisch: Krisenintervention}

Die Soziale Arbeit als „krisenbearbeitende Institution“ (Dollinger 2004, S. 382) nimmt in ihrer Zuschreibung von krisenhaften Seinszuständen durch ,gemeinsame Interpretation gesellschaftlicher Wirklichkeit“" (Dollinger 2004, S. 383) selbst normative Grenzziehungen vor. Welche Lebensweisen dabei als Gefährdende und als zu Intervenierende gelten, wird auch im Rahmen sozialpädagogischer Krisenintervention bei Kindeswohlgefährdung sichtbar. Dabei zeigt sich in der Rekonstruktion der sozialen Praktiken, dass - ebenso wie in der Untersuchung von Richter (2013) zur Sozialpädagogischen Familienhilfe - das institutionelle Setting durch ein „Ideal der bürgerlichen Kernfamilie als gesellschaftliche ,Messlatte' und (hetero)normative, geschlechtlich codierte Vergleichs- und Orientierungsfolie“ (Richter 2013, S. 15) strukturiert wird. Dadurch formen sich Normen „guter" Erziehung, welche durch die Idealisierung der bürgerlichen Kleinfamilie benachteiligte Familien gleichzeitig herabsetzen (vgl. Richter 2013, S. 16). In Dollingers Über- 
legungen zur Krisentheorie der Soziale Arbeit zeigt sich, dass das Bewältigungsverhalten nun genau auf die ,impliziten normativen Orientierungen aus[gerichtet wird], die in der Definition der Krise etabliert werden“"(Dollinger 2004, S. 393). Der folgende kleine Einblick in die Daten zeigt jedoch weniger den Aspekt des bürgerlichen Familienideals als vielmehr den Prozess der Grenzziehung in der Konstituierung von AdressatInnen als „gute“ oder „schlechte“ Eltern.

In der sozialpädagogischen Praxis hervorgebrachte und normative Grenzen lassen sich empirisch bspw. in Fallgeschichten rekonstruieren. Klatetzki (in Anlehnung an Star und Griesemer 1989, S. 393) sieht „Fallgeschichten der Sozialen Arbeit als idealtypische Grenzobjekte“ (Klatetzki 2013, S. 118), die zwischen verschiedenen sozialen Handlungsfeldern eine Verbindung herstellen (Klatetzki 2013, S. 117). Denn ,they have different meanings in different social worlds but their structure is common enough to more than one world to make them recognizable" (Star und Griesemer 1989, S. 393 zit. nach Klatetzki 2013, S. 118). In diesem Sinne können die folgenden Einblicke in empirisches Material als Fragmente einer Fallgeschichte verstanden werden, welche zwischen den zwei Handlungsfeldern Krisenintervention und Sozialbehörde fungieren.

Am Datenmaterial der einjährigen ethnographischen Feldphase in einer sozialpädagogischen Krisenintervention soll gezeigt werden, wie Elternschaft verhandelt wird und welche Grenzziehungen damit explizit und implizit verwoben sind. Wie dabei im pädagogischen Handeln normative Grenzen gezogen werden, wie Relationierungen des Sosein-sollens und des Nicht-so-Sein-sollens die Sozialpädagogik hervorbringen, das soll Gegenstand der Analyse sein. Die Grenzziehung zwischen einem krisenhaften Sein und einem idealen Sollen verdichtet sich am empirischen Material an der Grenze zwischen „guter" und „schlechter“ Elternschaft. Grenze wird damit zur Analysekategorie von Entscheidungs- und Legitimierungspraktiken.

Als Material dienen mir Auszüge aus einem Bericht über die vierjährige Ana, die Auskunft über die Betreuungsphase in der Zeit der Inobhutnahme gibt. Dieser Bericht entstand gegen Ende der sozialpädagogischen Krisenintervention vor dem Hintergrund der Verhandlung (und Legitimierung) über die weitere Unterbringung des Kindes. Die gewählten Auszüge zeigen die Konstituierung von Grenzen in Bezug auf Elternschaft besonders deutlich.

In einem ersten Abschnitt wird allgemein in Form eines professionellen pädagogischen Statements, wie mit Kinderschutzfällen in der sozialpädagogischen Krisenintervention umgegangen wird, geschrieben:

Wir versuchen den Kontakt zwischen Kind und primären Bezugspersonen nicht abbrechen zu lassen. Die Eltern sollen von Anfang an die Möglichkeit haben, ihr Kind regelmässig zu besuchen und/oder telefonisch mit ihm in Kontakt zu treten.

Bei kleinen Kindern sind tägliche Kontakte erwünscht.

Es geht um den normativen Anspruch, den Kontakt nicht abbrechen zu lassen und darum, ob die Eltern diesen erfüllen können oder nicht. Dies suggeriert eher eine passive Haltung der Professionellen. Sie sind abhängig von den primären Bezugspersonen in der Aufgabe, den Kontakt nicht abbrechen zu lassen. Dennoch wird diese Aufgabe als ein implizites Erfolgskriterium der Krisenintervention formuliert, was zugleich auf die Notwendigkeit der „produktiven“ Arbeit mit den Eltern verweist. Über den Inhalt oder die 
Qualität dieses Kontaktes wird nichts gesagt. Es geht um den Versuch, einen ,irgendwie gearteten Kontakt" nicht abbrechen zu lassen. Dass nicht hinterfragt wird, wie der Kontakt inhaltlich auszugestalten sei, verweist auf die implizite Annahme, dass jeglicher Kontakt von primären Bezugspersonen zum Kind als wichtig erachtet wird. In dieser Formulierung werden normative Vorstellungen eher unhinterfragt und inhaltsleer mitgetragen. Es lässt sich auch fragen, für wen der Kontaktversuch wichtig ist? Für die Professionellen, für die primären Bezugspersonen oder für das Kind selbst? Dahinter steht die implizite Annahme: Egal wie gut dieser Kontakt qualitativ ist, er hat immer eine hohe Bedeutung für das Kind und somit für das Kindeswohl. Die Annahme, der Kontakt zwischen Kind und primären Bezugspersonen sei zu fördern und möglichst zu versuchen, ihn nicht abbrechen zu lassen, führt gleichzeitig zur Immunisierung einer möglichen kritischen Haltung gegenüber kindeswohlgefährdenden Aspekten von Elternschaft. Man könnte auch sagen eine Idealisierung von Elternschaft bei gleichzeitiger Tabuisierung von Kindeswohlgefährdung und wiederholter Inszenierung von kindlicher Unmündigkeit. Ihm wird nämlich verunmöglicht, sich zu entziehen - es kann vor dem Hintergrund dieser normativen Setzung nicht über den Kontakt zu seinen primären Bezugspersonen mitentscheiden und seine Sichtweise wird hier ausgegrenzt. Dabei scheint es hier weniger um den Schutz des Kindes bzw. seines Wohls zu gehen, sondern viel eher um die Erfüllung einer normativen Hülle der Aufrechterhaltung von Kontakt. Es wird eine Grenze zwischen Eltern und Kind gezogen, die Eltern werden als aktiv und das Kind als passiv konstruiert, ihm wird Entscheidungsfreiheit genommen, es kommt nicht vor. Möglicherweise wird hier durch die Subjektivierung der Eltern, denen die Entscheidung überlassen wird, das Kind objektiviert. Zudem wird eine hierarchisierende Grenze zwischen primären und sekundären Bezugspersonen gezogen, denn wenn es primäre Bezugspersonen gibt, muss es auch sekundäre geben. Ebenso ist eine Verantwortungszuschreibung an die Eltern erkennbar; die Aufgabe der Professionellen bestünde so in der Aufforderung zur Zusicherung von Rahmenbedingungen (also Arrangieren von Kontaktmöglichkeit), nicht in der Zusicherung selbst.

Über die Mutter steht im Bericht:

Zur Mutter, obwohl wir uns bemühten, konnten wir keinen Kontakt herstellen. Die Mutter wusste, wo sich ihre Tochter befindet, machte aber nie den Versuch, sie dort sehen zu wollen. Ana traf während der Krisenintervention die Mutter ein paar Mal am Wochenende, das sie beim Vater oder der Grossmutter mütterlicherseits verbrachte. Den plötzlichen Beziehungsabbruch, Ende Jahr, beurteilen wir als traumatisches Erlebnis für Ana. Sie hat ab und zu, vor allem aber anfangs, nach der Mutter verlangt.

Es wird eine Art Verletzung von Professionsstolz sichtbar. Gegenüber der Mutter wird moralisierend und normativ argumentiert. Ihr wird unterstellt, dass sie nicht versucht hat $\mathrm{zu}$ wollen. Implizit steckt hier drin, dass eine Mutter zu wissen hat, wo ihre Tochter ist, ihre Tochter sehen zu wollen hat, und auch den Versuch zu unternehmen hat, sie zu sehen. Der als traumatisch beurteilte Beziehungsabbruch Ende Jahr, kurz bevor das Kind in Obhut genommen wurde, wirkt verstärkend moralisierend. In der weiteren Beschreibung hat das Kind dann nur manchmal, ab und zu und vor allem anfangs, nach der Mutter verlangt. Hier zeigen sich die normativ-moralische Hervorbringung einer Mutter und die 
Verantwortungsverschiebung der Professionellen auf die Mutter, welche zu wissen, zu wollen und zu tun hätte.

Über den Vater steht im Bericht:

Der Vater war die ganze Zeit eine feste, verlässliche Bezugsperson. [...] Der Vater äusserte anlässlich der ersten Sitzungen immer wieder wortgewaltig, wie er sein Leben auf Anas Bedürfnisse abstimmen werde. [...] Es zeigte sich, dass die Auflagen, die er und das fam. Umfeld erfüllen sollten, um Ana bei sich betreuen zu können, nicht erfüllt werden konnten. Der Vater war zwar verbal zu allem bereit, aber wenn es um die konkrete Umsetzung der Vorsätze ging, geschah nichts.

Die Zusammenarbeit mit dem Vater war angenehm, konfliktfrei. Er akzeptierte alles, opponierte nicht.

Obwohl der Vater zuerst als feste, verlässliche Bezugsperson eingeführt wird, lässt sich im weiteren Material ebendiese Verlässlichkeit seiner Tochter gegenüber dekonstruieren. Dies wirft die Frage auf, für wen der Vater denn als eine feste verlässliche Bezugsperson fungiert? Das Halten von Kontakt scheint auszureichen, um verlässlich zu sein und die ,angenehme, konfliktfreie, alles akzeptierende“ Zusammenarbeit des Vaters bewirkt, dass der Vater für die Professionellen zur festen verlässlichen, sich nicht widersetzenden Ansprechperson wird. Abgesehen von der als kritisch zu beurteilenden professionellen Deutungsweise, die hier nicht weiter ausgeführt werden soll, wird zwischen den Eltern eine Grenze gezogen: Der Vater wird zur ,guten“ Bezugsperson auch oder gerade weil die Mutter ja nicht erreichbar ist, obwohl sich die Professionellen bemühten. Das verstärkt nochmals die Wichtigkeit der Ansprechfunktion des Vaters für die Institution. Über den Ausschluss der Mutter als ,gute Mutter“ wird hier der Vater als „,verlässliche Bezugsperson“ und damit als ,guter Vater“ eingeschlossen. Die Konstruktion von Elternschaft geht einher mit verschiedenen normativen und auch moralischen Grenzziehungen. Mit Messmer (2012) kann „Moral als eine die institutionellen Aktivitäten der Sozialen Arbeit umfassende Orientierung“ (Messmer 2012, S. 17) verstanden werden, während deren explizite Thematisierung einerseits darauf abzielt, Kooperation oder auch Disziplinierung her- und sicherzustellen, andererseits Verhalten zu markieren, das institutionellen Erwartungen entgegenläuft (vgl. Messmer 2012, S. 18). „In diesem Fall wird die Verantwortung für das betreffende Verhalten explizit der Person zugerechnet. Dieser Situation liegt die Vorstellung eines ,guten“" Klienten zugrunde, der durch sein Verhalten die Absichten der Professionellen unterstützt" (Messmer, 2012, S. 18). Diese Argumentationsfigur findet sich in der Hervorbringung des Vaters als ,guter“ Vater, als verlässliche Ansprechperson für die Professionellen.

Zusammenfassend kann rekonstruiert werden: Innerhalb der Grenzen findet sich die leibliche und aktive Elternschaft sowie primäre Bezugspersonen, die normative Hülle der Aufrechterhaltung von Kontakt, die Quantität der Kontakte (anwesend sein als zentrales Kriterium), der Einschluss des Vaters als Anwesender, Verlässlicher und Verfügbarer, das Erhalten der Kernfamilie sowie eine Idealisierung von Elternschaft. Ausserhalb der Grenzziehung finden sich die soziale Elternschaft als sekundäre Bezugspersonen, die Qualität der Beziehung (im Gegensatz zur Quantität), der Ausschluss der Mutter als Abwesende, nicht Verlässliche und nicht Verfügbare, ebenso der Ausschluss des Kindes und seiner Perspektive, sowie eine Immunisierung von Kindeswohlgefährdung und der 
damit einhergehenden Entmündigung des Kindes sowie die Verantwortungsverschiebung von Professionellen zu Eltern. Die hier als Legitimierungsstrategien eingebrachten Grenzziehungen finden sich im weiteren Datenmaterial als normative Ordnungen, als strukturierte Begründungen, welche sich zur Rechtfertigung institutioneller Strukturen wie hier der sozialpädagogischen Krisenintervention - verdichten.

\section{Fazit}

Der Begriff der Grenze zeigt sich in seiner symbolischen und sozialen Bedeutung als gewinnbringende Perspektive für die sozialpädagogische Analyse ebenso wie für die professionelle Bearbeitung von Ungleichheiten. Denn über eine solcherart gefasste grenzanalytische Perspektive lässt sich auch die Normativität sozialpädagogischer Grenzziehungen in ihrer erkenntnistheoretischen und empirischen Wirkmächtigkeit herausarbeiten: Sozialpädagogische Kategorisierungen vermittels symbolischer Grenzen sowie die Bearbeitung ihrer Konsequenzen auf der Ebene sozialer Grenzen sind eingebunden in normative Ordnungen und bringen diese wiederum hervor. In der empirischen Rekonstruktion zeigen sich diese normativen Grenzziehungen in der Form von als plausibel akzeptierten Begründungen für sozialpädagogische Interventionen. Solche Rechtfertigungsnarrative oder Legitimierungsstrategien werden handlungsrelevant und konstituieren gleichsam soziale Wirklichkeiten in Form von Grenzverhältnissen. Insbesondere die für die Sozialpädagogik konstitutiven Annahmen ,guter Lebensführung“ wie beispielsweise (hetero)normative Vorstellungen von ,guter" Erziehung, ,guter“" Kindheit, „guter“ Familie und Elternschaft etc. korrelieren mit spezifischen Formen der professionellen Bearbeitung sozialpädagogischer Fälle. Neben der Reflexion der eigenen Beteiligung - als WissenschaftlerIn und ProfessionelleR - an Grenzziehungen ist es in diesem Sinne sozialpädagogische Aufgabe, Grenzen als verschiebbare offen zu legen und Soziale Arbeit als Grenzbearbeitung in den Blick zu nehmen (vgl. u. a. Heite et al. 2013; Kessl und Maurer 2010). Diese Praktiken der Grenzbearbeitung, Grenzsetzung, Grenzverschiebung lassen sich etwa hinsichtlich der Grenze zwischen Profession und Nicht-Profession (Heite 2013), der Erweiterung von Handlungsmöglichkeiten der AdressatInnen (Kessl 2009) oder am Beispiel von körper-/leibtheoretischen Analysen (Burghard et al. 2013 i. E.) justieren. Vor diesem Hintergrund zeigt sich die Grenze als analytische Perspektive wie Kategorie, welche die (Un)Möglichkeiten des (Anders-)Seins als kontingente und normativ plausibilisierte Ausschließungen erkennbar macht. Auf diese Weise kann eine grenzanalytische Perspektive sozialpädagogische Wirklichkeitskonstruktionen samt ihrer Konsequenzen in ihrer Bearbeitbarkeit offenlegen.

Open Access: Dieser Artikel unterliegt den Bedingungen der Creative Commons Attribution Noncommercial License. Dadurch sind die nichtkommerzielle Nutzung, Verteilung und Reproduktion erlaubt, sofern der/die Originalautor/en und die Quelle angegeben sind. 


\section{Literatur}

Burghard, A.-B., Magyar-Haas, V., \& Mörgen, R. (2013). Körper(leib)liche Dimensionen der Konstituierung von Grenzen. i. E.

Butler, J. (1995). Körper von Gewicht. Frankfurt a. M.: Suhrkamp.

Castel, R. (2008). Die Metamorphosen der sozialen Frage. Eine Chronik der Lohnarbeit. Konstanz: UVK.

Dollinger, B. (2004). Krisenintervention als Aufgabe der Sozialen Arbeit. Zeitschrift für Sozialpädagogik, 2(4), 377-396.

Dollinger, B. (2008). Sozialpädagogische Theorie zwischen Analyse und Zeitdiagnose. Widersprüche, (108), 31-42.

Donzelot, J. (1980). Die Ordnung der Familie. Frankfurt a. M.: Suhrkamp.

Forst, R., \& Günther, K. (2011). Die Herausbildung normativer Ordnungen. Zur Idee eines interdisziplinären Forschungsprogramms. In R. Forst \& K. Günther (Hrsg.), Die Herausbildung normativer Ordnungen (S. 11-30).

Heite, C. (2013). Setting and crossing boundaries: Professionalization of social work and social work professionalism. 1-14. In social work and society - special issue Social Work as Working at the Border. http://www.socwork.net/sws/index. Frankfurt a. M.: Campus.

Heite, C., Kessl, F., \& Maurer, S. (2013). Social work and society-special issue Social Work as Working at the Border. http://www.socwork.net/sws/index.

Kessl, F. (2009). Soziale Arbeit als Grenzbearbeiterin. Einige grenzanalytische Vergewisserungen. In S. Neumann \& P. Sandermann (Hrsg.), Kultur und Bildung. Neue Fluchtpunkte für die sozialpädagogische Forschung? (S. 154-169). Wiesbaden: VS Verlag für Sozialwissenschaften.

Kessl, F., \& Maurer, S. (2010). Praktiken der Differenzierung als Praktiken der Grenzbearbeitung. Überlegungen zur Bestimmung Sozialer Arbeit als Grenzbearbeiterin. In F. Kessl \& M. Plößer (Hrsg.), Differenzierung, Normalisierung, Andersheit. Soziale Arbeit als Arbeit mit den Anderen (S. 154-169). Wiesbaden: VS Verlag für Sozialwissenschaften.

Klatetzki, T. (2013). Die Fallgeschichte als Grenzobjekt. In R. Hörster, S. Köngeter, \& B. Müller (Hrsg.), Grenzobjekte. Soziale Welten und ihre Übergänge (S. 117-135). Wiesbaden: Springer.

Lamont, M., \& Molnár, V. (2002). The Study of Boundaries in the Social Sciences. Annual Review of Sociology, (28), 167-195.

Messmer, H. (2012). Moralstrukturen professionellen Handelns. In Soziale Passagen, 4(1), S. 5-22.

Richter, M. (2013). Die Sichtbarmachung des Familialen. Gesprächspraktiken in der Sozialpädagogischen Familienhilfe. Weinheim: Beltz Juventa.

Schäfer, A. (1989). Zur Kritik pädagogischer Wirklichkeitsentwürfe: Möglichkeiten und Grenzen pädagogischer Realitätsansprüche. Weinheim: Deutscher Studien Verlag.

Schäfer, A. (2012). Möglichkeitsräume: Von der Rhetorizität des Sozialen und dem polemischen Einsatz des Bildungsversprechens. Vierteljahresschrift für Wissenschaftliche Pädagogik, (4), 659-679.

Star, S. L., \& Griesemer, J. R. (1989). Institutional ecology, „Translation“ and boundary objects. Amateurs and professionals in Berkeley's museum of vertebrate zoology, 1907-1939. Social Studies of Science, 19(3), S. 387-420.

Wallace, R. J. (2011). Konzeptionen der Normativität: Einige grundlegende philosophische Fragen. In R. Forst \& K. Günther (Hrsg.), Die Herausbildung normativer Ordnungen (S. 33-55). Frankfurt a. M.: Campus Verlag. 